\title{
ON THE ZEROS OF EPSTEIN ZETA FUNCTIONS NEAR THE CRITICAL LINE
}

\author{
YOONBOK LEE
}

\begin{abstract}
Let $Q$ be a positive definite quadratic form with integral coefficients and let $E(s, Q)$ be the Epstein zeta function associated with $Q$. Assume that the class number of $Q$ is bigger than 1 . Then we estimate the number of zeros of $E(s, Q)$ in the region $\Re s>\sigma_{T}(\theta):=1 / 2+(\log T)^{-\theta}$ and $T<\Im s<2 T$, to provide its asymptotic formula for fixed $0<\theta<1$ conditionally. Moreover, it is unconditional if the class number of $Q$ is 2 or 3 and $0<\theta<1 / 13$.
\end{abstract}

\section{INTRODUCTION}

Let $K=\mathbb{Q}(\sqrt{D})$ be a quadratic imaginary field of class number $h:=h_{D}$ and let $\chi_{1}, \ldots, \chi_{h}$ be its ideal class characters. The Hecke $L$-function attached to $\chi_{j}$ is defined by

$$
L_{j}(s):=L\left(s, \chi_{j}\right):=\sum_{\mathfrak{n}} \frac{\chi_{j}(\mathfrak{n})}{\mathcal{N}(\mathfrak{n})^{s}}=\prod_{\mathfrak{p}}\left(1-\frac{\chi_{j}(\mathfrak{p})}{\mathcal{N}(\mathfrak{p})^{s}}\right)^{-1}
$$

for $\Re s>1$, where $\mathcal{N}$ is the norm. Each $L_{j}$ has an analytic continuation to $\mathbb{C}$ except for a possible pole at $s=1$ and it satisfies the functional equation

$$
\left(\frac{\sqrt{-D}}{2 \pi}\right)^{s} \Gamma(s) L_{j}(s)=\left(\frac{\sqrt{-D}}{2 \pi}\right)^{1-s} \Gamma(1-s) L_{j}(1-s) .
$$

By the Euler product and (1.1), $L_{j}$ has no zeros in $\Re s>1$ and the negative integers are the only zeros of $L_{j}$ in $\Re s<0$. All the other zeros are on the strip $0 \leq \Re s \leq 1$ and we believe that they are actually on the line $\Re s=1 / 2$.

These Hecke $L$-functions have a functional relation with an Epstein zeta function. To be precise, let $Q$ be a positive definite quadratic form with integral coefficients and its fundamental discriminant $D$. The Epstein zeta function $E(s, Q)$ associated with $Q$ is defined by

for $\Re s>1$. It satisfies

$$
E(s, Q):=\sum_{\substack{m, n \in \mathbb{Z} \\(m, n) \neq(0,0)}} \frac{1}{Q(m, n)^{s}}
$$

$$
E(s, Q)=\frac{w_{D}}{h_{D}} \sum_{j} \overline{\chi_{j}\left(\mathfrak{a}_{Q}\right)} L_{j}(s),
$$

where $w_{D}$ is the number of roots of unity in $K$ and $\mathfrak{a}_{Q}$ is an integer ideal in the ideal class corresponding to the equivalence class of $Q$. If $h_{D}=1$, then the Epstein zeta function $E(s, Q)=w_{D} L_{1}(s)$ is nothing but a Hecke $L$-function up to a constant factor. Hence we expect $E(s, Q)$ satisfy the generalized Riemann hypothesis. However, if $h_{D}>1$, the distribution of zeros of $E(s, Q)$ is different to the Riemann zeta function and indeed $E(s, Q)$

Date: November 9, 2018. 
has zeros off the line $\Re s=1 / 2$. Davenport and Heilbronn [3] showed that $E(s, Q)$ has infinitely many zeros on $\Re s>1$. Voronin [11] showed that the number of zeros of $E(s, Q)$ in the rectangle $\sigma_{1}<\Re s<\sigma_{2}$ and $T<\Im s<2 T$ is

$$
N_{E(s, Q)}\left(\sigma_{1}, \sigma_{2}: T, 2 T\right) \gg T
$$

for any fixed $1 / 2<\sigma_{1}<\sigma_{2}<1$ as a consequence of a joint universality of Hecke $L$-functions. The author in [8] proved that

$$
\lim _{T \rightarrow \infty} \frac{1}{T} N_{E(s, Q)}\left(\sigma_{1}, \sigma_{2}: T, 2 T\right)=\int_{\sigma_{1}}^{\sigma_{2}} g(\sigma) d \sigma
$$

holds for any $1 / 2<\sigma_{1} \leq \sigma_{2}$ and some nonnegative continuous function $g(\sigma)$. By a straightforward adaptation of [7], the author in [9] improved the above asymptotic formula to

$$
\frac{1}{T} N_{E(s, Q)}\left(\sigma_{1}, \sigma_{2}: T, 2 T\right)=\int_{\sigma_{1}}^{\sigma_{2}} g(\sigma) d \sigma+O\left(\frac{\log \log T}{(\log T)^{\sigma_{1} / 2}}\right)
$$

for $h=2,3$ and fixed $1 / 2<\sigma_{1}<\sigma_{2}<1$. The author with Gonek in [4] considered the case $h>3$ and proved that

$$
\frac{1}{T} N_{E(s, Q)}\left(\sigma_{1}, \sigma_{2}: T, 2 T\right)=\int_{\sigma_{1}}^{\sigma_{2}} g(\sigma) d \sigma+O\left(e^{-b \sqrt{\log \log T}}\right)
$$

for fixed $1 / 2<\sigma_{1}<\sigma_{2}<1$.

Now we examine the zero-density on or near the 1/2-line for a linear combination of $L$ functions with a same functional equation, which generalizes our Epstein zeta functions. Bombieri and Hejhal in 2] proved that almost all zeros of a linear combination $F(s)$ of inequivalent $L$-functions with a same functional equation are simple and on the $1 / 2$-line assuming RH and a zero-spacing assumption for each $L$-function. Hejhal in [6] investigated the zeros of $F(s)=(\cos \alpha) e^{i w_{1}} L_{1}(s)+(\sin \alpha) e^{i w_{2}} L_{2}(s)$ near the 1/2-line and showed that

$$
\frac{T \log T}{G \sqrt{\log \log T}} \ll N_{F(s)}\left(\sigma_{1}, \infty: T, 2 T\right) \ll \frac{T \log T}{G \sqrt{\log \log T}}
$$

for $\sigma_{1}=1 / 2+G / \log T$ and for almost all $\alpha$, where $(\log \log T)^{\kappa} \leq G \leq(\log T)^{1-\delta}, \kappa \in(1,3)$ and $\delta \in(0,1 / 10)$. We expect that it holds for all $\alpha$ except for the cases $\cos \alpha=0$ and $\sin \alpha=0$, but it is still an open question whether a given Epstein zeta function with class number 2 or 3 satisfy (1.3). Selberg in [10] sketched his idea which proves (1.3) for almost all linear combinations $F(s)$ of $L$-functions.

The aim of this paper is finding an asymptotic formula for the zero counting function

$$
N_{E(s, Q)}\left(\sigma_{T}, \infty: T, 2 T\right)
$$

of a given Epstein zeta function $E(s, Q)$ as $T \rightarrow \infty$, where

$$
\sigma_{T}=\sigma_{T}(\theta)=\frac{1}{2}+\frac{1}{(\log T)^{\theta}}
$$

for fixed $0<\theta<1$. Let $L_{1}, \ldots, L_{J}$ be inequivalent Hecke $L$-functions on $K$, i.e., $L_{j}(s)=$ $L\left(s, \chi_{j}\right)$ and $\chi_{j} \neq \chi_{\ell}, \bar{\chi}_{\ell}$ for $j \neq \ell$. By the Euler product, we may write

$$
\log L\left(s, \chi_{j}\right)=\sum_{p} \sum_{n=1}^{\infty} \frac{a_{j}\left(p^{n}\right)}{p^{n s}}
$$


for $\Re s>1$, then it is well-known that

$$
\sum_{p} \frac{a_{j}(p) a_{\ell}(p)}{p^{2 \sigma_{T}}}=\delta_{j, \ell} \xi_{j} \theta \log \log T+c_{j, \ell}+O\left(\frac{1}{(\log T)^{\theta}}\right)
$$

for $j, \ell \leq J$, where $\delta_{j, \ell}=1$ if $j=\ell, \delta_{j, \ell}=0$ if $j \neq \ell, \xi_{j}=4$ if $\chi_{j}$ is real, $\xi_{j}=2$ if $\chi_{j}$ is nonreal and the $c_{j, \ell}$ are some constants. Consider

$$
F_{J}(s):=\sum_{j=1}^{J} b_{j} L_{j}(s)
$$

for $b_{1}, \ldots, b_{J} \in \mathbb{C} \backslash\{0\}$ satisfying

$$
\left|b_{1}\right|^{2}+\cdots+\left|b_{J}\right|^{2}=1
$$

The Epstein zeta function $E(s, Q)$ is a special case of $F_{J}(s)$ up to a constant factor by (1.2) and the relation $L(s, \chi)=L(s, \bar{\chi})$. In this case, $J$ is the sum of the number of real characters and the half of the number of non-real characters. Hence, $J=2$ if $h=2,3$ and $J>2$ if $h>3$.

By Littlewood's lemma, the zero counting function

$$
N_{F_{J}}(\sigma: T):=N_{F_{J}}(\sigma, \infty: T, 2 T)
$$

for $\sigma>1 / 2$ is essentially a derivative of the integral

$$
\frac{1}{2 \pi} \int_{T}^{2 T} \log \left|F_{J}(\sigma+i t)\right| d t
$$

Moreover, we believe that the following conjecture is true.

Conjecture 1.1. Let $J>1,0<\theta<1$ and $\sigma_{T}=1 / 2+(\log T)^{-\theta}$. Then there exists $\eta>0$ such that

$$
\frac{1}{T} \int_{T}^{2 T} \log \left|F_{J}\left(\sigma_{T}+i t\right)\right| d t=\mathbb{E}\left[\log \left|F_{J}\left(\sigma_{T}: X\right)\right|\right]+O\left(\frac{1}{(\log T)^{\eta}}\right)
$$

as $T \rightarrow \infty$, where

$$
F_{J}(s: X):=\sum_{j=1}^{J} b_{j} L_{j}(s: X)
$$

and

$$
L_{j}(s: X):=\prod_{\mathfrak{p}}\left(1-\frac{\chi_{j}(\mathfrak{p}) X(\mathcal{N}(\mathfrak{p}))}{\mathcal{N}(\mathfrak{p})^{s}}\right)^{-1}
$$

are the random models of $F_{J}(s)$ and $L_{j}(s)$ for $j=1, \ldots, J$. Here, the $X(p)$ are uniformly and independently distributed on the unit circle $\mathbb{T}$ and $X\left(p^{\ell}\right):=X(p)^{\ell}$.

Conjecture 1.1 for $J>2$ is technically more difficult than the estimates in [4] for fixed $\sigma>1 / 2$, since there are more logarithmic singularties near the $1 / 2$-line. However, if $J=2$, it is possible to prove Conjecture 1.1 for a small $\theta$. One sees that

$$
\int_{T}^{2 T} \log \left|F_{2}\left(\sigma_{T}+i t\right)\right| d t=\int_{T}^{2 T} \log \left|b_{1} L_{2}\left(\sigma_{T}+i t\right)\right| d t+\int_{T}^{2 T} \log \left|\frac{L_{1}}{L_{2}}\left(\sigma_{T}+i t\right)+\frac{b_{2}}{b_{1}}\right| d t .
$$


The first integral on the right hand side can be estimated by an usual Dirichlet polynomial approximation for $\log \left|L_{2}\left(\sigma_{T}+i t\right)\right|$ and the second integral can be estimated by adapting [5]. Since its proof is straightforward from [5], we state it without a proof as follows.

Theorem 1.2. Conjecture 1.1 holds for $J=2$ and $0<\theta<1 / 13$ with $\eta<(1-13 \theta) / 4$.

The main feature of this paper is our estimation of

$$
\mathbb{E}\left[\log \left|F_{J}\left(\sigma_{T}: X\right)\right|\right]
$$

as $T \rightarrow \infty$ for $J>1$.

Theorem 1.3. Let $J>1,0<\theta<1$ and $\sigma_{T}=1 / 2+(\log T)^{-\theta}$. Then

$$
\begin{aligned}
\mathbb{E}\left[\log \left|F_{J}\left(\sigma_{T}: X\right)\right|\right]= & \frac{\sqrt{\theta \log \log T}}{\sqrt{\xi \pi^{J}}} \sum_{\ell=1}^{J} \int_{\mathcal{R}_{\ell}} u_{\ell} e^{-\sum_{j} u_{j}^{2} / \xi_{j}} d \boldsymbol{u} \\
& +\sum_{\ell=1}^{J} \frac{\log \left|b_{\ell}\right|}{\sqrt{\xi \pi^{J}}} \int_{\mathcal{R}_{\ell}} e^{-\sum_{j} u_{j}^{2} / \xi_{j}} d \boldsymbol{u}+O\left(\frac{1}{(\log \log T)^{1 / 4}}\right)
\end{aligned}
$$

as $T \rightarrow \infty$, where

$$
\begin{gathered}
\xi:=\prod_{j} \xi_{j}, \\
\mathcal{R}_{\ell}:=\left\{\left(u_{1}, \ldots, u_{J}\right) \in \mathbb{R}^{J}: u_{\ell}=\max \left\{u_{1}, \ldots, u_{J}\right\}\right\}
\end{gathered}
$$

for $\ell=1,2, \ldots, J$ and $d \boldsymbol{u}=d u_{1} \cdots d u_{J}$.

One expects that Littlewood's lemma, Conjecture 1.1 and Theorem 1.3 imply an asymptotic of $N_{F_{J}}\left(\sigma_{T}: T\right)$ as $T \rightarrow \infty$, but the $O$-term in Theorem 1.3 is too big. Instead, we estimate the difference

$$
\mathbb{E}\left[\log \left|F_{J}\left(\sigma_{T}\left(\theta_{1}\right): X\right)\right|\right]-\mathbb{E}\left[\log \left|F_{J}\left(\sigma_{T}\left(\theta_{2}\right): X\right)\right|\right]
$$

for a small $\left|\theta_{1}-\theta_{2}\right|$ to prove our main theorem.

Theorem 1.4. Let $J \geq 2$ and $0<\theta<1$ and assume Conjecture 1.1, then

$$
N_{F_{J}}\left(\sigma_{T}(\theta): T\right)=\frac{1}{4 \pi^{1+J / 2}} \frac{T(\log T)^{\theta}}{\sqrt{\xi \theta \log \log T}} \sum_{\ell=1}^{J} \int_{\mathcal{R}_{\ell}} u_{\ell} e^{-\sum_{j} u_{j}^{2} / \xi_{j}} d \boldsymbol{u}+O\left(\frac{T(\log T)^{\theta}}{(\log \log T)^{5 / 4}}\right)
$$

as $T \rightarrow \infty$, where $\xi$ and $\mathcal{R}_{\ell}$ are defined in Theorem 1.3.

By Theorem 1.2, we see that Theorem 1.4 holds for $J=2$ and $0<\theta<1 / 13$ unconditionally. When $F_{J}(s)$ is the Epstein zeta function $E(s, Q)$ up to a constant factor, it is interesting to see that

$$
\xi=2^{3 J-h}
$$

\section{Proof of Theorems 1.3 and 1.4}

Let

$$
\mathbf{L}(\sigma: X):=\left(\log \left|L_{1}(\sigma: X)\right|, \ldots, \log \left|L_{J}(\sigma: X)\right|, \Im \log L_{1}(\sigma: X), \ldots, \Im \log L_{J}(\sigma: X)\right) .
$$

Define

$$
\Psi_{\theta, T}(\mathbf{B}):=\mathbb{P}\left(\mathbf{L}\left(\sigma_{T}: X\right) \in \mathbf{B}\right)=\operatorname{meas}\left\{X \in \mathbb{T}^{\infty}: \mathbf{L}\left(\sigma_{T}: X\right) \in \mathbf{B}\right\}
$$


for a Borel set $\mathbf{B}$ in $\mathbb{R}^{2 J}$. It is known that the measure $\Psi_{\theta, T}$ has a density function $G_{\theta, T}$, so that for

$$
\Psi_{\theta, T}(\mathbf{B})=\int_{\mathbf{B}} G_{\theta, T}(\boldsymbol{u}, \boldsymbol{v}) d \boldsymbol{u} d \boldsymbol{v}
$$

where $\boldsymbol{u}=\left(u_{1}, \ldots, u_{J}\right), \boldsymbol{v}=\left(v_{1}, \ldots, v_{J}\right) \in \mathbb{R}^{J}$. (For instance, see the proof of Lemma 3.3 in [4.) Then we see that

$$
\begin{aligned}
\mathbb{E}\left[\log \left|F_{J}\left(\sigma_{T}: X\right)\right|\right] & =\int_{\mathbb{R}^{2 J}} \log \left|\sum_{j \leq J} b_{j} e^{u_{j}+i v_{j}}\right| d \Psi_{\theta, T}(\boldsymbol{u}, \boldsymbol{v}) \\
& =\int_{\mathbb{R}^{2 J}} \log \left|\sum_{j \leq J} b_{j} e^{u_{j}+i v_{j}}\right| G_{\theta, T}(\boldsymbol{u}, \boldsymbol{v}) d \boldsymbol{u} d \boldsymbol{v}
\end{aligned}
$$

and

$$
\hat{\Psi}_{\theta, T}(\boldsymbol{x}, \boldsymbol{y})=\int_{\mathbb{R}^{J}} e^{2 \pi i(\boldsymbol{u} \cdot \boldsymbol{x}+\boldsymbol{v} \cdot \boldsymbol{y})} d \Psi_{\theta, T}(\boldsymbol{u}, \boldsymbol{v})=\int_{\mathbb{R}^{J}} e^{2 \pi i(\boldsymbol{u} \cdot \boldsymbol{x}+\boldsymbol{v} \cdot \boldsymbol{y})} G_{\theta, T}(\boldsymbol{u}, \boldsymbol{v}) d \boldsymbol{u} d \boldsymbol{v}
$$

where $\boldsymbol{u}=\left(u_{1}, \ldots, u_{J}\right), \boldsymbol{v}=\left(v_{1}, \ldots, v_{J}\right), \boldsymbol{x}=\left(x_{1}, \ldots, x_{J}\right), \boldsymbol{y}=\left(y_{1}, \ldots, y_{J}\right) \in \mathbb{R}^{J}, \boldsymbol{u} \cdot \boldsymbol{x}=$ $u_{1} x_{1}+\cdots+u_{J} x_{J}$ and $\boldsymbol{v} \cdot \boldsymbol{y}=v_{1} y_{1}+\cdots+v_{J} y_{J}$. Since $G_{\theta, T}(\boldsymbol{u}, \boldsymbol{v})$ is the inverse Fourier transform of $\hat{\Psi}_{\theta, T}(\boldsymbol{x}, \boldsymbol{y})$

$$
G_{\theta, T}(\boldsymbol{u}, \boldsymbol{v})=\int_{\mathbb{R}^{J}} e^{-2 \pi i(\boldsymbol{u} \cdot \boldsymbol{x}+\boldsymbol{v} \cdot \boldsymbol{y})} \hat{\Psi}_{\theta, T}(\boldsymbol{x}, \boldsymbol{y}) d \boldsymbol{u} d \boldsymbol{v},
$$

we examine various properties of $\hat{\Psi}_{\theta, T}(\boldsymbol{x}, \boldsymbol{y})$ to study $G_{\theta, T}(\boldsymbol{u}, \boldsymbol{v})$.

Proposition 2.1. There exist constants $c_{1}, c_{2}>0$ such that

$$
\left|\hat{\Psi}_{\theta, T}(\boldsymbol{x}, \boldsymbol{y})\right| \leq \exp \left(-c_{1} \frac{\left(\sum_{j} x_{j}^{2}+y_{j}^{2}\right)^{1 / 2 \sigma_{T}}}{\log \left(\sum_{j} x_{j}^{2}+y_{j}^{2}\right)}\right)
$$

for $\sum_{j} x_{j}^{2}+y_{j}^{2} \geq c_{2}$,

$$
\left|\hat{\Psi}_{\theta, T}(\boldsymbol{x}, \boldsymbol{y})\right| \leq \exp \left(-\left(\frac{\pi^{2} \theta}{2} \log \log T+O(1)\right) \sum_{j \leq J}\left(x_{j}^{2}+y_{j}^{2}\right)\right)
$$

for $\sum_{j \leq J}\left(x_{j}^{2}+y_{j}^{2}\right) \leq e^{(\log T)^{\theta / 2}}$, and

$$
\hat{\Psi}_{\theta, T}(\boldsymbol{x}, \boldsymbol{y})=e^{-\pi^{2} \theta \log \log T \sum_{j \leq J} \xi_{j}\left(x_{j}^{2}+y_{j}^{2}\right)}\left(P(\boldsymbol{x}, \boldsymbol{y})+O\left(\sum_{j=1}^{J}\left(x_{j}^{2}+y_{j}^{2}\right)^{3}+\frac{1}{(\log T)^{\theta}}\right)\right)
$$

for $\sum_{j} x_{j}^{2}+y_{j}^{2} \leq c_{2}$, where

$$
P(\boldsymbol{x}, \boldsymbol{y}):=\sum_{\boldsymbol{k}, \ell \in\left(\mathbb{Z}_{\geq 0}\right)^{J}} \tilde{B}_{\boldsymbol{k}, \ell} \boldsymbol{x}^{\boldsymbol{k}} \boldsymbol{y}^{\ell}
$$

and the coefficients $\tilde{B}_{\boldsymbol{k}, \boldsymbol{\ell}}$ are independent to $\theta$ and $T$ satisfying

$$
\tilde{B}_{0,0}=1
$$

and

$$
\tilde{B}_{k, \ell}=0
$$


if $\mathcal{K}(\boldsymbol{k}+\boldsymbol{\ell})=1$ or $>5$. Here, $\boldsymbol{x}=\left(x_{1}, \ldots, x_{J}\right), \boldsymbol{y}=\left(y_{1}, \ldots, y_{J}\right) \in \mathbb{R}^{J}, \boldsymbol{k}=\left(k_{1}, \ldots, k_{J}\right), \boldsymbol{\ell}=$ $\left(\ell_{1}, \ldots, \ell_{J}\right) \in\left(\mathbb{Z}_{\geq 0}\right)^{J}$ and

$$
\mathcal{K}(\boldsymbol{k}):=k_{1}+\cdots+k_{J}, \quad \boldsymbol{x}^{\boldsymbol{k}}:=\prod_{j \leq J} x_{j}^{k_{j}} .
$$

Proposition 2.2. Let $\boldsymbol{k}, \boldsymbol{\ell}, \boldsymbol{m}, \boldsymbol{n}$ be vectors in $\left(\mathbb{Z}_{\geq 0}\right)^{J}$ and

$$
q_{\boldsymbol{k}, \ell: \boldsymbol{m}, \boldsymbol{n}}=\frac{\tilde{B}_{2 \boldsymbol{k}+\boldsymbol{m}, 2 \boldsymbol{\ell}+\boldsymbol{n}}}{i^{\mathcal{K}(\boldsymbol{m}+\boldsymbol{n})} \pi^{2 J+\mathcal{K}(2 \boldsymbol{k}+2 \boldsymbol{\ell}+\boldsymbol{m}+\boldsymbol{n})}} \prod_{j}\left(\frac{\Gamma\left(k_{j}+1 / 2\right) \Gamma\left(\ell_{j}+1 / 2\right)}{\xi_{j}^{k_{j}+\ell_{j}+m_{j}+n_{j}+1}}\right) \frac{(2 \boldsymbol{k}+\boldsymbol{m}) !(2 \boldsymbol{\ell}+\boldsymbol{n}) !}{(2 \boldsymbol{k}) ! \boldsymbol{m} !(2 \boldsymbol{\ell}) ! \boldsymbol{n} !} .
$$

Then, we have

$$
\begin{aligned}
G_{\theta, T}(\boldsymbol{u}, \boldsymbol{v})= & \exp \left(-\sum_{j} \frac{u_{j}^{2}+v_{j}^{2}}{\theta \xi_{j} \log \log T}\right) \sum_{\boldsymbol{k}, \boldsymbol{\ell}, \boldsymbol{m}, \boldsymbol{n} \in\left(\mathbb{Z}_{\geq 0}\right)^{J}} \frac{q_{\boldsymbol{k}, \boldsymbol{\ell} \boldsymbol{m}, \boldsymbol{n}}}{(\theta \log \log T)^{J+\mathcal{K}(\boldsymbol{k}+\boldsymbol{\ell}+\boldsymbol{m}+\boldsymbol{n})}} \boldsymbol{u}^{\boldsymbol{m}} \boldsymbol{v}^{\boldsymbol{n}} \\
& +O\left(\frac{1}{(\log \log T)^{J+3}}\right)
\end{aligned}
$$

for all $\boldsymbol{u}=\left(u_{1}, \ldots, u_{J}\right), \boldsymbol{v}=\left(v_{1}, \ldots, v_{J}\right) \in \mathbb{R}^{J}$, and there exists a constant $c>0$ such that

$$
G_{\theta, T}(\boldsymbol{u}, \boldsymbol{v}) \ll \exp \left(-c \sum_{j \leq J} \frac{u_{j}^{2}+v_{j}^{2}}{\log \log T}\right)
$$

for all $\boldsymbol{u}=\left(u_{1}, \ldots, u_{J}\right), \boldsymbol{v}=\left(v_{1}, \ldots, v_{J}\right) \in \mathbb{R}^{J}$.

Note that

$$
q_{0,0: 0,0}=\pi^{-J} \prod_{j} \xi_{j}^{-1}=\pi^{-J} \xi^{-1}
$$

and

$$
q_{\boldsymbol{k}, \ell: m, n}=0
$$

if $\mathcal{K}(2 \boldsymbol{k}+2 \boldsymbol{\ell}+\boldsymbol{m}+\boldsymbol{n})=1$ or $>5$. We also need the following lemma.

Lemma 2.3. Let $k$ be a positive integer, $M \geq 1$ and $b_{j} \in \mathbb{C}$ for $j \leq J$. Then there exists an absolute constant $C>0$ such that

$$
\int_{\mathbb{R}^{2 J}}|\log | \sum_{j \leq J} b_{j} e^{u_{j}+i v_{j}}||^{2 k} e^{-\sum_{j}\left(u_{j}^{2}+v_{j}^{2}\right) / M} d \boldsymbol{u} d \boldsymbol{v} \ll M^{J+k}(C k)^{k}+M^{J}(C k)^{2 k} .
$$

We prove the propositions and the lemma in 93 . Now we shall estimate the integral in (2.2). By the Cauchy-Schwarz inequality, Proposition 2.2 and Lemma 2.3, we find that

$$
\mathbb{E}\left[\log \left|F_{J}\left(\sigma_{T}: X\right)\right|\right]=\int_{\left[-M_{1}, M_{1}\right]^{2 J}} \log \left|\sum_{j \leq J} b_{j} e^{u_{j}+i v_{j}}\right| G_{\theta, T}(\boldsymbol{u}, \boldsymbol{v}) d \boldsymbol{u} d \boldsymbol{v}+O\left(\frac{1}{(\log \log T)^{\eta}}\right)
$$

for any $\eta>0$, where $M_{1}=\eta^{\prime} \sqrt{\log \log T \log \log \log T}$ with $\eta^{\prime}>0$ depending on $\eta$. By the Cauchy-Schwarz inequality and Lemma 2.3, we also have

$$
\begin{aligned}
\left(\int_{\left[-M_{1}, M_{1}\right]^{2 J}} \log \left|\sum_{j \leq J} b_{j} e^{u_{j}+i v_{j}}\right| d \boldsymbol{u} d \boldsymbol{v}\right)^{2} \\
\leq\left(2 M_{1}\right)^{2 J} \int_{\left[-M_{1}, M_{1}\right]^{2 J}}\left(\log \left|\sum_{j \leq J} b_{j} e^{u_{j}+i v_{j}}\right|\right)^{2} d \boldsymbol{u} d \boldsymbol{v}
\end{aligned}
$$




$$
\begin{aligned}
& \ll M_{1}^{2 J} \int_{\mathbb{R}^{2 J}}\left(\log \left|\sum_{j \leq J} b_{j} e^{u_{j}+i v_{j}}\right|\right)^{2} e^{-\sum_{j}\left(u_{j}^{2}+v_{j}^{2}\right) / M_{1}^{2}} d \boldsymbol{u} d \boldsymbol{v} \\
& \ll M_{1}^{4 J+2} .
\end{aligned}
$$

Hence, by Proposition 2.2

$$
\begin{aligned}
\mathbb{E}\left[\log \left|F_{J}\left(\sigma_{T}: X\right)\right|\right] & \sum_{\boldsymbol{k}, \boldsymbol{\ell}, \boldsymbol{m}, \boldsymbol{n} \in\left(\mathbb{Z}_{\geq 0}\right)^{J}} \frac{q_{\boldsymbol{k}, \boldsymbol{\ell}: \boldsymbol{m}, \boldsymbol{n}}}{(\theta \log \log T)^{J+K(\boldsymbol{k}+\boldsymbol{\ell}+\boldsymbol{m}+\boldsymbol{n})}} \\
& \quad \int_{\left[-M_{1}, M_{1}\right]^{2 J}} \log \left|\sum_{j \leq J} b_{j} e^{u_{j}+i v_{j}}\right| \exp \left(-\sum_{j} \frac{u_{j}^{2}+v_{j}^{2}}{\theta \xi_{j} \log \log T}\right) \boldsymbol{u}^{\boldsymbol{m}} \boldsymbol{v}^{\boldsymbol{n}} d \boldsymbol{u} d \boldsymbol{v} \\
& +O\left(\frac{(\log \log \log T)^{J+1 / 2}}{(\log \log T)^{5 / 2}}\right) .
\end{aligned}
$$

Once again, by the Cauchy-Schwarz inequality, Proposition 2.2 and Lemma 2.3, we find that $\mathbb{E}\left[\log \left|F_{J}\left(\sigma_{T}: X\right)\right|\right]$

$$
\begin{aligned}
= & \sum_{\boldsymbol{k}, \boldsymbol{\ell}, \boldsymbol{m}, \boldsymbol{n} \in\left(\mathbb{Z}_{\geq 0}\right)^{J}} \frac{q_{\boldsymbol{k}, \boldsymbol{\ell} \boldsymbol{m}, \boldsymbol{n}}}{(\theta \log \log T)^{J+\mathcal{K}(\boldsymbol{k}+\boldsymbol{\ell}+\boldsymbol{m}+\boldsymbol{n})}} \\
& \int_{\mathbb{R}^{2 J}} \log \left|\sum_{j \leq J} b_{j} e^{u_{j}+i v_{j}}\right| \exp \left(-\sum_{j} \frac{u_{j}^{2}+v_{j}^{2}}{\theta \xi_{j} \log \log T}\right) \boldsymbol{u}^{\boldsymbol{m}} \boldsymbol{v}^{\boldsymbol{n}} d \boldsymbol{u} d \boldsymbol{v} \\
& +O\left(\frac{(\log \log \log T)^{J+1 / 2}}{(\log \log T)^{5 / 2}}\right) \\
= & \sum_{\boldsymbol{k}, \boldsymbol{\ell}, \boldsymbol{m}, \boldsymbol{n} \in\left(\mathbb{Z}_{\geq 0}\right)^{J}} \frac{q_{\boldsymbol{k}, \boldsymbol{\ell} \boldsymbol{m}, \boldsymbol{n}}}{(\theta \log \log T)^{\mathcal{K}(\boldsymbol{k}+\boldsymbol{\ell})+\mathcal{K}(\boldsymbol{m}+\boldsymbol{n}) / 2}} I_{\boldsymbol{m}, \boldsymbol{n}}(\theta, T)+O\left(\frac{(\log \log \log T)^{J+1 / 2}}{(\log \log T)^{5 / 2}}\right),
\end{aligned}
$$

where

$$
I_{\boldsymbol{m}, \boldsymbol{n}}(\theta, T):=\int_{\mathbb{R}^{2 J}} \log \left|\sum_{j \leq J} b_{j} e^{\left(u_{j}+i v_{j}\right) \sqrt{\theta \log \log T}}\right| e^{-\sum_{j}\left(u_{j}^{2}+v_{j}^{2}\right) / \xi_{j}} \boldsymbol{u}^{\boldsymbol{m}} \boldsymbol{v}^{\boldsymbol{n}} d \boldsymbol{u} d \boldsymbol{v} .
$$

The logarithm is dominated by the biggest term in the $j$-sum, so that we divide $\mathbb{R}^{2 J}$ into $J$ pieces

$$
I_{\boldsymbol{m}, \boldsymbol{n}}(\theta, T)=\sum_{\ell=1}^{J} \int_{\mathbb{R}^{J}} \int_{\mathcal{R}_{\ell}} \log \left|\sum_{j \leq J} b_{j} e^{\left(u_{j}+i v_{j}\right) \sqrt{\theta \log \log T}}\right| e^{-\sum_{j}\left(u_{j}^{2}+v_{j}^{2}\right) / \xi_{j}} \boldsymbol{u}^{\boldsymbol{m}} \boldsymbol{v}^{\boldsymbol{n}} d \boldsymbol{u} d \boldsymbol{v},
$$

where $\mathcal{R}_{\ell}$ is defined in (1.6). By symmetry, it is enough to consider $\mathcal{R}_{1}$. Then

$$
\begin{aligned}
\int_{\mathbb{R}^{J}} \int_{\mathcal{R}_{1}} \log \left|\sum_{j \leq J} b_{j} e^{\left(u_{j}+i v_{j}\right) \sqrt{\theta \log \log T}}\right| e^{-\sum_{j}\left(u_{j}^{2}+v_{j}^{2}\right) / \xi_{j}} \boldsymbol{u}^{\boldsymbol{m}} \boldsymbol{v}^{\boldsymbol{n}} d \boldsymbol{u} d \boldsymbol{v} \\
\quad=\int_{\mathbb{R}^{J}} \int_{\mathcal{R}_{1}} \log \left|b_{1} e^{\left(u_{1}+i v_{1}\right) \sqrt{\theta \log \log T}}\right| e^{-\sum_{j}\left(u_{j}^{2}+v_{j}^{2}\right) / \xi_{j}} \boldsymbol{u}^{\boldsymbol{m}} \boldsymbol{v}^{\boldsymbol{n}} d \boldsymbol{u} d \boldsymbol{v}+\mathcal{E}_{\boldsymbol{m}, \boldsymbol{n}, 1}(\theta, T) \\
=d_{\boldsymbol{n}} \int_{\mathcal{R}_{1}}\left(\sqrt{\theta \log \log T} u_{1}+\log \left|b_{1}\right|\right) e^{-\sum_{j} u_{j}^{2} / \xi_{j}} \boldsymbol{u}^{\boldsymbol{m}} d \boldsymbol{u}+\mathcal{E}_{\boldsymbol{m}, \boldsymbol{n}, 1}(\theta, T),
\end{aligned}
$$


where

$$
\mathcal{E}_{\boldsymbol{m}, \boldsymbol{n}, 1}(\theta, T):=\int_{\mathbb{R}^{J}} \int_{\mathcal{R}_{1}} \log \left|1+\sum_{j=2}^{J} \frac{b_{j}}{b_{1}} e^{\left(u_{j}-u_{1}+i\left(v_{j}-v_{1}\right)\right) \sqrt{\theta \log \log T}}\right| e^{-\sum_{j}\left(u_{j}^{2}+v_{j}^{2}\right) / \xi_{j}} \boldsymbol{u}^{\boldsymbol{m}} \boldsymbol{v}^{\boldsymbol{n}} d \boldsymbol{u} d \boldsymbol{v}
$$

and

$$
d_{\boldsymbol{n}}:=\int_{\mathbb{R}^{J}} e^{-\sum_{j} v_{j}^{2} / \xi_{j}} \boldsymbol{v}^{n} d \boldsymbol{v}=\prod_{j \leq J}\left(\xi_{j}^{\left(n_{j}+1\right) / 2} \int_{\mathbb{R}} v^{n_{j}} e^{-v^{2}} d v\right) .
$$

Note that $\int_{\mathbb{R}} v^{n_{j}} e^{-v^{2}} d v=0$ if $n_{j}$ is odd, and $=\Gamma\left(\left(n_{j}+1\right) / 2\right)$ otherwise. Therefore,

$$
\begin{aligned}
I_{\boldsymbol{m}, \boldsymbol{n}}(\theta, T)= & \sum_{\ell=1}^{J} d_{\boldsymbol{n}} \int_{\mathcal{R}_{\ell}}\left(\sqrt{\theta \log \log T} u_{\ell}+\log \left|b_{\ell}\right|\right) e^{-\sum_{j} u_{j}^{2} / \xi_{j}} \boldsymbol{u}^{\boldsymbol{m}} d \boldsymbol{u}+\mathcal{E}_{\boldsymbol{m}, \boldsymbol{n}}(\theta, T) \\
= & \sqrt{\theta \log \log T} d_{\boldsymbol{n}} \sum_{\ell=1}^{J} \int_{\mathcal{R}_{\ell}} u_{\ell} e^{-\sum_{j} u_{j}^{2} / \xi_{j}} \boldsymbol{u}^{\boldsymbol{m}} d \boldsymbol{u} \\
& +d_{\boldsymbol{n}} \sum_{\ell=1}^{J} \int_{\mathcal{R}_{\ell}} \log \left|b_{\ell}\right| e^{-\sum_{j} u_{j}^{2} / \xi_{j}} \boldsymbol{u}^{\boldsymbol{m}} d \boldsymbol{u}+\mathcal{E}_{\boldsymbol{m}, \boldsymbol{n}}(\theta, T),
\end{aligned}
$$

where

$$
\mathcal{E}_{\boldsymbol{m}, \boldsymbol{n}}(\theta, T):=\sum_{\ell=1}^{J} \mathcal{E}_{\boldsymbol{m}, \boldsymbol{n}, \ell}(\theta, T) .
$$

By estimating $\mathcal{E}_{\boldsymbol{m}, \boldsymbol{n}}(\theta, T)$ in $\$ 3.4$, we prove the following proposition.

Proposition 2.4. Let $J \geq 2$. Then we have

$$
\mathcal{E}_{\boldsymbol{m}, \boldsymbol{n}}(\theta, T)=O\left(\frac{1}{(\log \log T)^{1 / 4}}\right)
$$

Therefore, by (2.3), (2.5) and Propositions 2.4 and 2.2 we find that

$$
\begin{aligned}
\mathbb{E} & {\left[\log \left|F_{J}\left(\sigma_{T}: X\right)\right|\right] } \\
= & q_{0,0: 0,0} d_{0}\left(\sqrt{\theta \log \log T} \sum_{\ell=1}^{J} \int_{\mathcal{R}_{\ell}} u_{\ell} e^{-\sum_{j} u_{j}^{2} / \xi_{j}} d \boldsymbol{u}+\sum_{\ell=1}^{J} \int_{\mathcal{R}_{\ell}} \log \left|b_{\ell}\right| e^{-\sum_{j} u_{j}^{2} / \xi_{j}} d \boldsymbol{u}\right) \\
& +\sum_{\mathcal{K}(\boldsymbol{m})=1} q_{0,0: \boldsymbol{m}, 0} d_{0} \sum_{\ell=1}^{J} \int_{\mathcal{R}_{\ell}} u_{\ell} e^{-\sum_{j} u_{j}^{2} / \xi_{j}} \boldsymbol{u}^{\boldsymbol{m}} d \boldsymbol{u}+O\left(\frac{1}{(\log \log T)^{1 / 4}}\right) \\
= & \pi^{-J / 2} \prod_{j} \xi_{j}^{-1 / 2} \sqrt{\theta \log \log T} \sum_{\ell=1}^{J} \int_{\mathcal{R}_{\ell}} u_{\ell} e^{-\sum_{j} u_{j}^{2} / \xi_{j}} d \boldsymbol{u} \\
& +\pi^{-J / 2} \prod_{j} \xi_{j}^{-1 / 2} \sum_{\ell=1}^{J} \log \left|b_{\ell}\right| \int_{\mathcal{R}_{\ell}} e^{-\sum_{j} u_{j}^{2} / \xi_{j}} d \boldsymbol{u}+O\left(\frac{1}{(\log \log T)^{1 / 4}}\right) .
\end{aligned}
$$

This proves Theorem 1.3 . 
Next we prove Theorem 1.4 assuming Conjecture 1.1. By Littlewood's lemma and (2.3) we see that

$$
\begin{aligned}
\int_{\sigma_{T}\left(\theta_{1}\right)}^{\sigma_{T}\left(\theta_{2}\right)} & N_{F_{J}}(w: T) d w \\
= & \frac{1}{2 \pi} \int_{T}^{2 T} \log \left|F_{J}\left(\sigma_{T}\left(\theta_{1}\right)+i t\right)\right| d t \\
& -\frac{1}{2 \pi} \int_{T}^{2 T} \log \left|F_{J}\left(\sigma_{T}\left(\theta_{2}\right)+i t\right)\right| d t+O\left(\frac{T}{(\log T)^{\theta_{2}}}\right) \\
= & \frac{T}{2 \pi}\left(\mathbb{E}\left[\log \left|F_{J}\left(\sigma_{T}\left(\theta_{1}\right): X\right)\right|\right]-\mathbb{E}\left[\log \left|F_{J}\left(\sigma_{T}\left(\theta_{2}\right): X\right)\right|\right]\right)+O\left(\frac{T}{(\log T)^{\eta}}\right),
\end{aligned}
$$

where $\sigma_{T}(\theta)=1 / 2+(\log T)^{-\theta}$ and $0<\theta_{2}<\theta_{1}$. We need the following lemma.

Lemma 2.5. Let $\alpha$ be a real number, $\theta_{1}>\theta_{2}>0$ and $H_{T}=\theta_{1}-\theta_{2}$. Suppose that $H_{T} \rightarrow 0$ as $T \rightarrow \infty$. Then for each $i=1,2$ we have

$$
\begin{aligned}
\theta_{1}^{\alpha} I_{\boldsymbol{m}, \boldsymbol{n}}\left(\theta_{1}, T\right)-\theta_{2}^{\alpha} I_{\boldsymbol{m}, \boldsymbol{n}}\left(\theta_{2}, T\right) \\
=H_{T} \sqrt{\log \log T} d_{\boldsymbol{n}}\left(\alpha+\frac{1}{2}\right) \theta_{i}^{\alpha-1 / 2} \sum_{\ell=1}^{J} \int_{\mathcal{R}_{\ell}} u_{\ell} e^{-\sum_{j} u_{j}^{2} / \xi_{j}} \boldsymbol{u}^{m} d \boldsymbol{u} \\
\quad+H_{T} d_{\boldsymbol{n}} \alpha \theta_{i}^{\alpha-1} \sum_{\ell=1}^{J} \int_{\mathcal{R}_{\ell}} \log \left|b_{\ell}\right| e^{-\sum_{j} u_{j}^{2} / \xi_{j}} \boldsymbol{u}^{\boldsymbol{m}} d \boldsymbol{u} \\
+ \\
+O\left(\frac{H_{T}}{(\log \log T)^{1 / 4}}+H_{T}^{2} \sqrt{\log \log T}\right) .
\end{aligned}
$$

We prove it in 93.5 . Suppose that $H_{T} \log \log T=o(1)$, then

$$
\begin{aligned}
\int_{\sigma_{T}(\theta)}^{\sigma_{T}\left(\theta-H_{T}\right)} N_{F_{J}}(w: T) d w & \leq\left(\sigma_{T}\left(\theta-H_{T}\right)-\sigma_{T}(\theta)\right) N_{F_{J}}\left(\sigma_{T}(\theta): T\right) \\
& =\frac{H_{T} \log \log T}{(\log T)^{\theta}}\left(1+O\left(H_{T} \log \log T\right)\right) N_{F_{J}}\left(\sigma_{T}(\theta): T\right)
\end{aligned}
$$

and

$$
\begin{aligned}
\int_{\sigma_{T}\left(\theta+H_{T}\right)}^{\sigma_{T}(\theta)} N_{F_{J}}(w: T) d w & \geq\left(\sigma_{T}(\theta)-\sigma_{T}\left(\theta+H_{T}\right)\right) N_{F_{J}}\left(\sigma_{T}(\theta): T\right) \\
& =\frac{H_{T} \log \log T}{(\log T)^{\theta}}\left(1+O\left(H_{T} \log \log T\right)\right) N_{F_{J}}\left(\sigma_{T}(\theta): T\right) .
\end{aligned}
$$

By (2.7), (2.3) and Lemma 2.5, we find that

$$
\begin{aligned}
\frac{H_{T} \log \log T}{(\log T)^{\theta}} & \left(1+O\left(H_{T} \log \log T\right)\right) N_{F_{J}}\left(\sigma_{T}(\theta): T\right) \\
= & \frac{H_{T} T}{2 \pi} \sum_{\boldsymbol{k}, \ell, \boldsymbol{m}, \boldsymbol{n} \in\left(\mathbb{Z}_{\geq 0}\right)^{J}} \frac{q_{\boldsymbol{k}, \boldsymbol{\ell}: \boldsymbol{m}, \boldsymbol{n}}}{(\theta \log \log T)^{\mathcal{K}(\boldsymbol{k}+\boldsymbol{\ell})+\mathcal{K}(\boldsymbol{m}+\boldsymbol{n}) / 2}}
\end{aligned}
$$




$$
\begin{aligned}
& \left(\sqrt{\log \log T} d_{\boldsymbol{n}}\left(-\mathcal{K}(\boldsymbol{k}+\boldsymbol{\ell})+\frac{1-\mathcal{K}(\boldsymbol{m}+\boldsymbol{n})}{2}\right) \theta^{-1 / 2} \sum_{\ell=1}^{J} \int_{\mathcal{R}_{\ell}} u_{\ell} e^{-\sum_{j} u_{j}^{2} / \xi_{j}} \boldsymbol{u}^{\boldsymbol{m}} d \boldsymbol{u}\right. \\
& \left.+d_{\boldsymbol{n}}\left(-\mathcal{K}(\boldsymbol{k}+\boldsymbol{\ell})-\frac{\mathcal{K}(\boldsymbol{m}+\boldsymbol{n})}{2}\right) \theta^{-1} \sum_{\ell=1}^{J} \int_{\mathcal{R}_{\ell}} \log \left|b_{\ell}\right| e^{-\sum_{j} u_{j}^{2} / \xi_{j}} \boldsymbol{u}^{\boldsymbol{m}} d \boldsymbol{u}\right) \\
& +O\left(\frac{T(\log \log \log T)^{J+1 / 2}}{(\log \log T)^{5 / 2}}+\frac{H_{T} T}{(\log \log T)^{1 / 4}}+H_{T}^{2} T \sqrt{\log \log T}\right) .
\end{aligned}
$$

Choose $H_{T}=(\log \log T)^{-2}$ to optimize it, we see that

$$
\begin{aligned}
N_{F_{J}}\left(\sigma_{T}(\theta): T\right)= & \frac{T(\log T)^{\theta}}{2 \pi} \sum_{\boldsymbol{k}, \ell, \boldsymbol{m}, \boldsymbol{n} \in\left(\mathbb{Z}_{\geq 0}\right)^{J}} \frac{q_{\boldsymbol{k}, \boldsymbol{\ell}: \boldsymbol{m}, \boldsymbol{n}} d_{\boldsymbol{n}}}{(\theta \log \log T)^{1+\mathcal{K}(\boldsymbol{k}+\boldsymbol{\ell})+\mathcal{K}(\boldsymbol{m}+\boldsymbol{n}) / 2}} \\
& \left(\sqrt{\theta \log \log T}\left(-\mathcal{K}(\boldsymbol{k}+\boldsymbol{\ell})+\frac{1-\mathcal{K}(\boldsymbol{m}+\boldsymbol{n})}{2}\right) \sum_{\ell=1}^{J} \int_{\mathcal{R}_{\ell}} u_{\ell} e^{-\sum_{j} u_{j}^{2} / \xi_{j}} \boldsymbol{u}^{\boldsymbol{m}} d \boldsymbol{u}\right. \\
& \left.+\left(-\mathcal{K}(\boldsymbol{k}+\boldsymbol{\ell})-\frac{\mathcal{K}(\boldsymbol{m}+\boldsymbol{n})}{2}\right) \sum_{\ell=1}^{J} \int_{\mathcal{R}_{\ell}} \log \left|b_{\ell}\right| e^{-\sum_{j} u_{j}^{2} / \xi_{j}} \boldsymbol{u}^{\boldsymbol{m}} d \boldsymbol{u}\right) \\
& +O\left(\frac{T(\log T)^{\theta}}{(\log \log T)^{5 / 4}}\right) .
\end{aligned}
$$

We see that the summands are smaller than the $O$-term unless $\boldsymbol{k}=\boldsymbol{\ell}=0$ and $\mathcal{K}(\boldsymbol{m}+\boldsymbol{n})=$ 0,1 . Moreover, $q_{0,0: \boldsymbol{m}, \boldsymbol{n}}=0$ if $\mathcal{K}(\boldsymbol{m}+\boldsymbol{n})=1$. Hence,

$$
N_{F_{J}}\left(\sigma_{T}(\theta): T\right)=\frac{T(\log T)^{\theta}}{\sqrt{\theta \log \log T}} \frac{q_{0,0: 0,0} d_{0}}{4 \pi} \sum_{\ell=1}^{J} \int_{\mathcal{R}_{\ell}} u_{\ell} e^{-\sum_{j} u_{j}^{2} / \xi_{j}} d \boldsymbol{u}+O\left(\frac{T(\log T)^{\theta}}{(\log \log T)^{5 / 4}}\right) .
$$

Since

$$
q_{0,0: 0,0} d_{0}=\pi^{-J / 2} \prod_{j} \xi_{j}^{-1 / 2}
$$

we prove the theorem.

\section{Proof of PROPOSITIONS AND LEMMAS}

3.1. Proof of Proposition 2.1. Let $z_{j}=\pi\left(x_{j}+i y_{j}\right)$ for $j=1, \ldots, J$, then

$$
\begin{aligned}
\hat{\Psi}_{\theta, T}(\boldsymbol{x}, \boldsymbol{y}) & =\mathbb{E}\left[\exp \left(2 \pi i \sum_{j \leq J}\left(x_{j} \Re \log L_{j}\left(\sigma_{T}: X\right)+y_{j} \Im \log L_{j}\left(\sigma_{T}: X\right)\right)\right)\right] \\
& =\mathbb{E}\left[\exp \left(2 \pi i \sum_{j \leq J} \Re\left[\left(x_{j}-i y_{j}\right) \log L_{j}\left(\sigma_{T}: X\right)\right]\right)\right] \\
& =\mathbb{E}\left[\exp \left(i \sum_{j \leq J} \bar{z}_{j} \log L_{j}\left(\sigma_{T}: X\right)+z_{j} \log L_{j}\left(\sigma_{T}: \bar{X}\right)\right)\right] .
\end{aligned}
$$

Write

$$
\log L_{j}(\sigma: X):=\sum_{p} g_{j}(p, \sigma: X), \quad g_{j}(p, \sigma: X):=\sum_{n=1}^{\infty} \frac{a_{j}\left(p^{n}\right) X(p)^{n}}{p^{n \sigma}}
$$


then

$$
\begin{aligned}
\hat{\Psi}_{\theta, T}(\boldsymbol{x}, \boldsymbol{y}) & =\prod_{p} \mathbb{E}\left[\exp \left(i \sum_{j \leq J} \bar{z}_{j} g_{j}\left(p, \sigma_{T}: X\right)+z_{j} g_{j}\left(p, \sigma_{T}: \bar{X}\right)\right)\right] \\
& =\prod_{p} \mathbb{E}\left[\prod_{j \leq J} \exp \left(i\left(\bar{z}_{j} g_{j}\left(p, \sigma_{T}: X\right)+z_{j} g_{j}\left(p, \sigma_{T}: \bar{X}\right)\right)\right)\right]
\end{aligned}
$$

and we see that

$$
\left|\mathbb{E}\left[\prod_{j \leq J} \exp \left(i\left(\bar{z}_{j} g_{j}\left(p, \sigma_{T}: X\right)+z_{j} g_{j}\left(p, \sigma_{T}: \bar{X}\right)\right)\right)\right]\right| \leq 1 .
$$

By Lemma 2.5 in [8] and the argument to justify the equation (3.28) in [8, p. $1828-1829]$, there is a constant $C_{1}>0$ such that

$$
\left|\mathbb{E}\left[\prod_{j \leq J} \exp \left(i\left(\bar{z}_{j} g_{j}\left(p, \sigma_{T}: X\right)+z_{j} g_{j}\left(p, \sigma_{T}: \bar{X}\right)\right)\right)\right]\right| \leq C_{1} \frac{p^{\sigma_{T} / 2}}{\left(\sum_{j} x_{j}^{2}+y_{j}^{2}\right)^{1 / 4}}
$$

for $p^{-\sigma_{T}} \sqrt{\sum_{j} x_{j}^{2}+y_{j}^{2}} \geq 1$. Hence if $p^{\sigma_{T}} \leq C_{2} \sqrt{\sum_{j} x_{j}^{2}+y_{j}^{2}}$ with $C_{2}=\min \left\{1, C_{1}^{-2} e^{-1}\right\}$, then

$$
\left|\mathbb{E}\left[\prod_{j \leq J} \exp \left(i\left(\bar{z}_{j} g_{j}\left(p, \sigma_{T}: X\right)+z_{j} g_{j}\left(p, \sigma_{T}: \bar{X}\right)\right)\right)\right]\right| \leq e^{-1 / 2} .
$$

Thus, by (3.1), (3.2) and the prime number theorem, we have

$$
\left|\hat{\Psi}_{\theta, T}(\boldsymbol{x}, \boldsymbol{y})\right| \leq \prod_{p^{\sigma_{T}} \leq C_{2} \sqrt{\sum_{j} x_{j}^{2}+y_{j}^{2}}} e^{-1 / 2} \leq \exp \left(-C_{3} \frac{\left(\sum_{j} x_{j}^{2}+y_{j}^{2}\right)^{1 / 2 \sigma_{T}}}{\log \left(\sum_{j} x_{j}^{2}+y_{j}^{2}\right)}\right)
$$

for $\sum_{j} x_{j}^{2}+y_{j}^{2} \geq C_{4}$ and for some $C_{3}, C_{4}>0$. This proves the first inequality in Proposition 2.1 .

Let

$$
A_{k, \ell}(p, \sigma):=\mathbb{E}\left[\prod_{j \leq J} g_{j}(p, \sigma: X)^{k_{j}} g_{j}(p, \sigma: \bar{X})^{\ell_{j}}\right]
$$

for $\boldsymbol{k}=\left(k_{1}, \ldots, k_{J}\right)$ and $\boldsymbol{\ell}=\left(\ell_{1}, \ldots, \ell_{J}\right)$, then each factor of $\hat{\Psi}_{\theta, T}(\boldsymbol{x}, \boldsymbol{y})$ is

$$
\begin{aligned}
& \left.\sum_{\boldsymbol{k}, \ell \geq 0} \frac{i^{\mathcal{K}(\boldsymbol{k}+\ell)}}{\boldsymbol{k} ! \ell !} \mathbb{E}\left[\prod_{j \leq J} g_{j}\left(p, \sigma_{T}: X\right)^{k_{j}} g_{j}\left(p, \sigma_{T}: \bar{X}\right)^{\ell_{j}}\right)\right] \overline{\boldsymbol{z}}^{\boldsymbol{k}} \boldsymbol{z}^{\ell} \\
& =\sum_{\boldsymbol{k}, \boldsymbol{\ell} \geq 0} \frac{i^{\mathcal{K}(\boldsymbol{k}+\ell)}}{\boldsymbol{k} ! \ell !} A_{\boldsymbol{k}, \boldsymbol{\ell}}\left(p, \sigma_{T}\right) \overline{\boldsymbol{z}}^{\boldsymbol{k}} \boldsymbol{z}^{\boldsymbol{\ell}},
\end{aligned}
$$

where the sums are over all $\boldsymbol{k}=\left(k_{1}, \ldots, k_{J}\right), \boldsymbol{\ell}=\left(\ell_{1}, \ldots, \ell_{J}\right) \in\left(\mathbb{Z}_{\geq 0}\right)^{J}$ and $\mathcal{K}(\boldsymbol{k})=k_{1}+\cdots+$ $k_{J}, \boldsymbol{k} !=k_{1} ! \cdots k_{J} !$ and $\boldsymbol{z}^{\boldsymbol{k}}=\prod_{j \leq J} z_{j}^{k_{j}}$. Since $A_{0,0}(p, \sigma)=1$ and $A_{0, \boldsymbol{k}}(p, \sigma)=A_{\boldsymbol{k}, 0}(p, \sigma)=0$ for $\boldsymbol{k} \neq 0$, the above sum equals

$$
1+\sum_{\boldsymbol{k}, \ell}^{*} \frac{i^{\mathcal{K}(\boldsymbol{k}+\ell)}}{\boldsymbol{k} ! \ell !} A_{\boldsymbol{k}, \ell}\left(p, \sigma_{T}\right) \overline{\boldsymbol{z}}^{k} \boldsymbol{z}^{\ell}=: 1+\mathcal{A}_{\theta, T, \boldsymbol{x}, \boldsymbol{y}}(p)
$$


where the $*$-sum is over all nonzero $\boldsymbol{k}, \boldsymbol{\ell} \in\left(\mathbb{Z}_{\geq 0}\right)^{J}$. By estimating (3.3) one can show that

$$
\left|A_{\boldsymbol{k}, \ell}\left(p, \sigma_{T}\right)\right| \leq C_{5} p^{-\sigma_{T} \mathcal{K}(\boldsymbol{k}+\ell)}
$$

for some $C_{5}>0$ and all nonzero $\boldsymbol{k}, \boldsymbol{\ell} \in\left(\mathbb{Z}_{\geq 0}\right)^{J}$. Thus,

$$
\left|\mathcal{A}_{\theta, T, \boldsymbol{x}, \boldsymbol{y}}(p)\right| \leq C_{5} \sum_{\boldsymbol{k}, \ell}^{*} \frac{1}{\boldsymbol{k} ! \ell !} \prod_{j \leq J}\left(\frac{\left|z_{j}\right|}{p^{\sigma_{T}}}\right)^{k_{j}+\ell_{j}}=C_{5}\left(\exp \left(\frac{\sum_{j=1}^{J}\left|z_{j}\right|}{p^{\sigma_{T}}}\right)-1\right)^{2}
$$

Let $Y=e^{(\log T)^{\theta / 2}}$, then there exists a constant $C_{6}>0$ such that

$$
\left|\mathcal{A}_{\theta, T, \boldsymbol{x}, \boldsymbol{y}}(p)\right| \leq C_{6} \frac{\sum_{j=1}^{J}\left|z_{j}\right|^{2}}{p^{2 \sigma_{T}}} \leq C_{6}
$$

for $\sum_{j \leq J}\left|z_{j}\right|^{2} \leq Y$ and $p \geq Y$. Thus, by (3.1)

$$
\begin{aligned}
\left|\hat{\Psi}_{\theta, T}(\boldsymbol{x}, \boldsymbol{y})\right| & \leq\left|\prod_{p \geq Y}\left(1+\mathcal{A}_{\theta, T, \boldsymbol{x}, \boldsymbol{y}}(p)\right)\right| \\
& =\left|\prod_{p \geq Y} \exp \left(\mathcal{A}_{\theta, T, \boldsymbol{x}, \boldsymbol{y}}(p)+O\left(\frac{\left(\sum_{j=1}^{J}\left|z_{j}\right|^{2}\right)^{2}}{p^{4 \sigma_{T}}}\right)\right)\right| \\
& =\left|\exp \left(\sum_{p \geq Y} \mathcal{A}_{\theta, T, \boldsymbol{x}, \boldsymbol{y}}(p)+O\left(\sum_{j=1}^{J}\left|z_{j}\right|^{2}\right)\right)\right|
\end{aligned}
$$

The $p$-sum is

$$
\begin{aligned}
\sum_{p \geq Y} \mathcal{A}_{\theta, T, \boldsymbol{x}, \boldsymbol{y}}(p) & =\sum_{p \geq Y} \sum_{\boldsymbol{k}, \boldsymbol{\ell}} \frac{i^{\mathcal{K}(\boldsymbol{k}+\boldsymbol{\ell})}}{\boldsymbol{k} ! \boldsymbol{\ell} !} A_{\boldsymbol{k}, \boldsymbol{\ell}}\left(p, \sigma_{T}\right) \overline{\boldsymbol{z}}^{\boldsymbol{k}} \boldsymbol{z}^{\boldsymbol{\ell}} \\
& \left.=-\sum_{p \geq Y} \sum_{j_{1}, j_{2} \leq J} \mathbb{E}\left[g_{j_{1}}\left(p, \sigma_{T}: X\right) g_{j_{2}}\left(p, \sigma_{T}: \bar{X}\right)\right)\right] \bar{z}_{j_{1}} z_{j_{2}}+O\left(\sum_{j=1}^{J}\left|z_{j}\right|^{2}\right) \\
& =-\sum_{j_{1}, j_{2} \leq J} \sum_{p \geq Y} \frac{a_{j_{1}}(p) a_{j_{2}}(p)}{p^{2 \sigma_{T}}} \bar{z}_{j_{1}} z_{j_{2}}+O\left(\sum_{j=1}^{J}\left|z_{j}\right|^{2}\right) \\
& =-\sum_{j \leq J}\left|z_{j}\right|^{2}\left(\sum_{p \geq Y} \frac{a_{j}(p)^{2}}{p^{2 \sigma_{T}}}+O(1)\right) \\
& \leq-\left(\frac{\pi^{2} \theta}{2} \log \log T+O(1)\right) \sum_{j \leq J}\left(x_{j}^{2}+y_{j}^{2}\right) .
\end{aligned}
$$

Therefore,

$$
\left|\hat{\Psi}_{\theta, T}(\boldsymbol{x}, \boldsymbol{y})\right| \leq \exp \left(-\left(\frac{\pi^{2} \theta}{2} \log \log T+O(1)\right) \sum_{j \leq J}\left(x_{j}^{2}+y_{j}^{2}\right)\right)
$$

holds for $\sum_{j \leq J}\left(x_{j}^{2}+y_{j}^{2}\right) \leq e^{(\log T)^{\theta / 2}}$, which proves the second inequality in Proposition 2.1. 
Next, we find an asymptotic of $\hat{\Psi}_{\theta, T}(\boldsymbol{x}, \boldsymbol{y})$ for $\sum_{j \leq J}\left(x_{j}^{2}+y_{j}^{2}\right) \leq C_{7}$. By (3.4) and choosing $C_{7}>0$ sufficiently small, we have that $\left|\mathcal{A}_{\theta, T, \boldsymbol{x}, \boldsymbol{y}}(p)\right| \leq 1 / 2$ for every prime $p$. Thus,

$$
\begin{aligned}
\hat{\Psi}_{\theta, T}(\boldsymbol{x}, \boldsymbol{y}) & =\prod_{p}\left(1+\mathcal{A}_{\theta, T, \boldsymbol{x}, \boldsymbol{y}}(p)\right) \\
& =\prod_{p} \exp \left(\mathcal{A}_{\theta, T, \boldsymbol{x}, \boldsymbol{y}}(p)-\frac{1}{2}\left(\mathcal{A}_{\theta, T, \boldsymbol{x}, \boldsymbol{y}}(p)\right)^{2}+O\left(\frac{\sum_{j=1}^{J}\left(x_{j}^{2}+y_{j}^{2}\right)^{3}}{p^{6 \sigma_{T}}}\right)\right) \\
& =\exp \left(\sum_{p} \mathcal{A}_{\theta, T, \boldsymbol{x}, \boldsymbol{y}}(p)-\frac{1}{2} \sum_{p}\left(\mathcal{A}_{\theta, T, \boldsymbol{x}, \boldsymbol{y}}(p)\right)^{2}+O\left(\sum_{j=1}^{J}\left(x_{j}^{2}+y_{j}^{2}\right)^{3}\right)\right) .
\end{aligned}
$$

The sum

$$
\sum_{p} \mathcal{A}_{\theta, T, \boldsymbol{x}, \boldsymbol{y}}(p)-\frac{1}{2} \sum_{p}\left(\mathcal{A}_{\theta, T, \boldsymbol{x}, \boldsymbol{y}}(p)\right)^{2}
$$

has a power series representation in $z_{1}, \bar{z}_{1}, \ldots, z_{J}, \bar{z}_{J}$, so let it be

$$
\sum_{k, \ell}^{*} B_{k, \ell}\left(\sigma_{T}\right) \bar{z}^{k} z^{\ell}
$$

For $\mathcal{K}(\boldsymbol{k}+\boldsymbol{\ell}) \geq 3$, we have

$$
B_{\boldsymbol{k}, \ell}\left(\sigma_{T}\right)=B_{\boldsymbol{k}, \ell}(1 / 2)+O\left(\frac{1}{(\log T)^{\theta}}\right)
$$

For $\mathcal{K}(\boldsymbol{k})=\mathcal{K}(\ell)=1$, we have

$$
\begin{aligned}
\sum_{\mathcal{K}(\boldsymbol{k})=\mathcal{K}(\ell)=1} B_{\boldsymbol{k}, \ell}\left(\sigma_{T}\right) & =\sum_{p} \sum_{\mathcal{K}(\boldsymbol{k})=\mathcal{K}(\ell)=1} \frac{i^{\mathcal{K}(\boldsymbol{k}+\ell)}}{\boldsymbol{k} ! \ell !} A_{\boldsymbol{k}, \boldsymbol{\ell}}\left(p, \sigma_{T}\right) \overline{\boldsymbol{z}}^{\boldsymbol{k}} \boldsymbol{z}^{\boldsymbol{\ell}} \\
& =-\sum_{p} \sum_{j_{1}, j_{2} \leq J} \mathbb{E}\left[g_{j_{1}}\left(p, \sigma_{T}: X\right) g_{j_{2}}\left(p, \sigma_{T}: \bar{X}\right)\right] \bar{z}_{j_{1}} z_{j_{2}} .
\end{aligned}
$$

By (1.4), we find that

$$
\left.\mathbb{E}\left[g_{j_{1}}\left(p, \sigma_{T}: X\right) g_{j_{2}}\left(p, \sigma_{T}: \bar{X}\right)\right)\right]=C_{j_{1}, j_{2}}+O\left(\frac{1}{(\log T)^{\theta}}\right)
$$

for $j_{1} \neq j_{2}$ and

$$
\begin{aligned}
\left.\mathbb{E}\left[g_{j}\left(p, \sigma_{T}: X\right) g_{j}\left(p, \sigma_{T}: \bar{X}\right)\right)\right] & =\sum_{p} \frac{a_{j}(p)^{2}}{p^{2 \sigma_{T}}}+C_{j}^{\prime}+O\left(\frac{1}{(\log T)^{\theta}}\right) \\
& =\xi_{j} \theta \log \log T+C_{j, j}+O\left(\frac{1}{(\log T)^{\theta}}\right)
\end{aligned}
$$

for some constants $C_{j_{1}, j_{2}}, C_{j, j}, C_{j}^{\prime}$ independent to $\theta$. Thus,

$$
\sum_{\mathcal{K}(\boldsymbol{k})=\mathcal{K}(\ell)=1} B_{\boldsymbol{k}, \ell}\left(\sigma_{T}\right)=-\theta \log \log T \sum_{j \leq J} \xi_{j}\left|z_{j}\right|^{2}+\sum_{j_{1}, j_{2} \leq J} C_{j_{1}, j_{2}} \bar{z}_{j_{1}} z_{j_{2}}+O\left(\frac{1}{(\log T)^{\theta}}\right) .
$$


Therefore, we have

$$
\begin{aligned}
\hat{\Psi}_{\theta, T}(\boldsymbol{x}, \boldsymbol{y})= & e^{-\theta \log \log T \sum_{j \leq J} \xi_{j}\left|z_{j}\right|^{2}} \exp \left(\sum_{j_{1}, j_{2} \leq J} C_{j_{1}, j_{2}} \bar{z}_{j_{1}} z_{j_{2}}+\sum_{\mathcal{K}(\boldsymbol{k}+\ell)=3,4,5} B_{\boldsymbol{k}, \ell}(1 / 2) \overline{\boldsymbol{z}}^{\boldsymbol{k}} \boldsymbol{z}^{\ell}\right) \\
& \times \exp \left(O\left(\sum_{j=1}^{J}\left(x_{j}^{2}+y_{j}^{2}\right)^{3}+\frac{1}{(\log T)^{\theta}}\right)\right) \\
= & e^{-\pi^{2} \theta \log \log T \sum_{j \leq J} \xi_{j}\left(x_{j}^{2}+y_{j}^{2}\right)}\left(P(\boldsymbol{x}, \boldsymbol{y})+O\left(\sum_{j=1}^{J}\left(x_{j}^{2}+y_{j}^{2}\right)^{3}+\frac{1}{(\log T)^{\theta}}\right)\right)
\end{aligned}
$$

for $\sum_{j \leq J}\left(x_{j}^{2}+y_{j}^{2}\right) \leq C_{7}$, where $P(\boldsymbol{x}, \boldsymbol{y})$ is a polynomial of degree $\leq 5$ and may be written as

$$
1+\sum_{\mathcal{K}(\boldsymbol{k}+\ell)=2,3,4,5} \tilde{B}_{\boldsymbol{k}, \ell} \boldsymbol{x}^{\boldsymbol{k}} \boldsymbol{y}^{\ell}
$$

This completes the proof of Proposition 2.1.

3.2. Proof of Proposition 2.2, By Proposition 2.1, we have

$$
\begin{aligned}
G_{\theta, T}(\boldsymbol{u}, \boldsymbol{v})= & \int_{\mathbb{R}^{2 J}} \hat{\Psi}_{\theta, T}(\boldsymbol{x}, \boldsymbol{y}) e^{-2 \pi i(\boldsymbol{x} \cdot \boldsymbol{u}+\boldsymbol{y} \cdot \boldsymbol{v})} d \boldsymbol{x} d \boldsymbol{y} \\
= & \int_{\sum_{j}\left(x_{j}^{2}+y_{j}^{2}\right) \leq C_{7}} e^{-\pi^{2} \theta \log \log T \sum_{j \leq J} \xi_{j}\left(x_{j}^{2}+y_{j}^{2}\right)-2 \pi i(\boldsymbol{x} \cdot \boldsymbol{u}+\boldsymbol{y} \cdot \boldsymbol{v})} P(\boldsymbol{x}, \boldsymbol{y}) d \boldsymbol{x} d \boldsymbol{y} \\
& +O\left(\frac{1}{(\log \log T)^{J+3}}\right) \\
= & \int_{\mathbb{R}^{2 J}} e^{-\pi^{2} \theta \log \log T \sum_{j \leq J} \xi_{j}\left(x_{j}^{2}+y_{j}^{2}\right)-2 \pi i(\boldsymbol{x} \cdot \boldsymbol{u}+\boldsymbol{y} \cdot \boldsymbol{v})} P(\boldsymbol{x}, \boldsymbol{y}) d \boldsymbol{x} d \boldsymbol{y} \\
& +O\left(\frac{1}{(\log \log T)^{J+3}}\right),
\end{aligned}
$$

where $P(\boldsymbol{x}, \boldsymbol{y})$ is the polynomial defined in Proposition 2.1. By the change of variables

$$
x_{j}=\frac{\tilde{x}_{j}}{\pi \sqrt{\theta \xi_{j} \log \log T}}-\frac{i u_{j}}{\pi \theta \xi_{j} \log \log T}
$$

and

$$
y_{j}=\frac{\tilde{y}_{j}}{\pi \sqrt{\theta \xi_{j} \log \log T}}-\frac{i v_{j}}{\pi \theta \xi_{j} \log \log T}
$$

one finds that

$$
P(\boldsymbol{x}, \boldsymbol{y})=\sum_{\boldsymbol{k}, \ell, \boldsymbol{m}, \boldsymbol{n} \in\left(\mathbb{Z}_{\geq 0}\right)^{J}} \frac{p_{\boldsymbol{k}, \ell: \boldsymbol{m}, \boldsymbol{n}}}{(\theta \log \log T)^{\mathcal{K}(\boldsymbol{k}+\ell) / 2+\mathcal{K}(\boldsymbol{m}+\boldsymbol{n})}} \tilde{\boldsymbol{x}}^{\boldsymbol{k}} \tilde{\boldsymbol{y}}^{\ell} \boldsymbol{u}^{\boldsymbol{m}} \boldsymbol{v}^{\boldsymbol{n}}
$$

where

$$
p_{\boldsymbol{k}, \ell: \boldsymbol{m}, \boldsymbol{n}}=\frac{\tilde{B}_{\boldsymbol{k}+\boldsymbol{m}, \boldsymbol{\ell}+\boldsymbol{n}}}{\pi^{\mathcal{K}(\boldsymbol{k}+\ell+\boldsymbol{m}+\boldsymbol{n})} i^{\mathcal{K}(\boldsymbol{m}+\boldsymbol{n})}} \frac{(\boldsymbol{k}+\boldsymbol{m}) !(\boldsymbol{\ell}+\boldsymbol{n}) !}{\boldsymbol{k} ! \boldsymbol{m} ! \ell ! \boldsymbol{n} !} \prod_{j} \xi^{-\left(k_{j}+\ell_{j}\right) / 2-m_{j}-n_{j}}
$$

Then we see that

$$
G_{\theta, T}(\boldsymbol{u}, \boldsymbol{v})=\exp \left(-\sum_{j} \frac{u_{j}^{2}+v_{j}^{2}}{\theta \xi_{j} \log \log T}\right) \frac{1}{\prod_{j}\left(\pi^{2} \theta \xi_{j} \log \log T\right)}
$$




$$
\begin{aligned}
& \int_{\mathbb{R}^{2 J}} e^{-\sum_{j}\left(\tilde{x}_{j}^{2}+\tilde{y}_{j}^{2}\right)} \sum_{\boldsymbol{k}, \boldsymbol{\ell}, \boldsymbol{m}, \boldsymbol{n} \in\left(\mathbb{Z}_{\geq 0}\right)^{J}} \frac{p_{\boldsymbol{k}, \boldsymbol{\ell}: \boldsymbol{m}, \boldsymbol{n}}}{(\theta \log \log T)^{\mathcal{K}(\boldsymbol{k}+\ell) / 2+\mathcal{K}(\boldsymbol{m}+\boldsymbol{n})}} \tilde{\boldsymbol{x}}^{\boldsymbol{k}} \tilde{\boldsymbol{y}}^{\ell} \boldsymbol{u}^{\boldsymbol{m}} \boldsymbol{v}^{\boldsymbol{n}} d \tilde{\boldsymbol{x}} d \tilde{\boldsymbol{y}} \\
& +O\left(\frac{1}{(\log \log T)^{J+3}}\right) \\
= & \exp \left(-\sum_{j} \frac{u_{j}^{2}+v_{j}^{2}}{\theta \xi_{j} \log \log T}\right) \sum_{\boldsymbol{k}, \ell, \boldsymbol{m}, \boldsymbol{n} \in\left(\mathbb{Z}_{\geq 0}\right)^{J}} \frac{c_{\boldsymbol{k}, \ell} p_{\boldsymbol{k}, \ell: \boldsymbol{m}, \boldsymbol{n}}}{(\theta \log \log T)^{J+\mathcal{K}(\boldsymbol{k}+\boldsymbol{\ell}) / 2+\mathcal{K}(\boldsymbol{m}+\boldsymbol{n})}} \boldsymbol{u}^{\boldsymbol{m}} \boldsymbol{v}^{\boldsymbol{n}} \\
& +O\left(\frac{1}{(\log \log T)^{J+3}}\right),
\end{aligned}
$$

where

$$
\begin{aligned}
c_{\boldsymbol{k}, \boldsymbol{\ell}} & :=\pi^{-2 J}\left(\prod_{j} \xi_{j}^{-1}\right) \int_{\mathbb{R}^{2 J}} e^{-\sum_{j}\left(\tilde{x}_{j}^{2}+\tilde{y}_{j}^{2}\right)} \tilde{\boldsymbol{x}}^{k} \tilde{\boldsymbol{y}}^{\ell} d \tilde{\boldsymbol{x}} d \tilde{\boldsymbol{y}} \\
& =\pi^{-2 J} \prod_{j}\left(\xi_{j}^{-1} \int_{\mathbb{R}} e^{-x^{2}} x^{k_{j}} d x \int_{\mathbb{R}} e^{-y^{2}} y^{\ell_{j}} d y\right) .
\end{aligned}
$$

Thus, if there is odd $k_{j}$ or odd $\ell_{j}$, then $c_{\boldsymbol{k}, \ell}=0$. Otherwise,

$$
c_{\boldsymbol{k}, \ell}=\pi^{-2 J} \prod_{j}\left(\xi_{j}^{-1} \Gamma\left(\frac{k_{j}+1}{2}\right) \Gamma\left(\frac{\ell_{j}+1}{2}\right)\right) .
$$

Hence, we have

$$
\begin{aligned}
G_{\theta, T}(\boldsymbol{u}, \boldsymbol{v})= & \exp \left(-\sum_{j} \frac{u_{j}^{2}+v_{j}^{2}}{\theta \xi_{j} \log \log T}\right) \sum_{\boldsymbol{k}, \boldsymbol{\ell}, \boldsymbol{m}, \boldsymbol{n} \in\left(\mathbb{Z}_{\geq 0}\right)^{J}} \frac{c_{2 \boldsymbol{k}, 2 \ell} p_{2 \boldsymbol{k}, 2 \ell: \boldsymbol{m}, \boldsymbol{n}}}{(\theta \log \log T)^{J+\mathcal{K}(\boldsymbol{k}+\boldsymbol{\ell}+\boldsymbol{m}+\boldsymbol{n})}} \boldsymbol{u}^{\boldsymbol{m}} \boldsymbol{v}^{\boldsymbol{n}} \\
& +O\left(\frac{1}{(\log \log T)^{J+3}}\right) .
\end{aligned}
$$

Letting $q_{\boldsymbol{k}, \boldsymbol{\ell}: \boldsymbol{m}, \boldsymbol{n}}=c_{2 \boldsymbol{k}, 2 \ell} p_{2 \boldsymbol{k}, 2 \ell: \boldsymbol{m}, \boldsymbol{n}}$, we prove the first identity of the proposition. The second one can be deduced by modifying the proof of Theorem 6 in [1].

3.3. Proof of Lemma 2.3. Our proof is basically the same as the proof of Lemma 3.3 in [4], but we need the dependency on $M$. We first see that

$$
\begin{aligned}
\int_{\mathbb{R}^{2 J}}|\log | \sum_{j \leq J} b_{j} e^{u_{j}+i v_{j}}||^{2 k} e^{-\sum_{j}\left(u_{j}^{2}+v_{j}^{2}\right) / M} d \boldsymbol{u} d \boldsymbol{v} \\
=\int_{\mathbb{R}^{J}} \int_{[0,2 \pi]^{J}} \sum_{\boldsymbol{k} \in \mathbb{Z}^{J}}|\log | \sum_{j \leq J} b_{j} e^{u_{j}+i\left(v_{j}+2 \pi k_{j}\right)}||^{2 k} e^{-\sum_{j}\left(u_{j}^{2}+\left(v_{j}+2 \pi k_{j}\right)^{2}\right) / M} d \boldsymbol{v} d \boldsymbol{u} \\
=\int_{\mathbb{R}^{J}} \int_{[0,2 \pi]^{J}}|\log | \sum_{j \leq J} b_{j} e^{u_{j}+i v_{j}}||^{2 k} \sum_{\boldsymbol{k} \in \mathbb{Z}^{J}} e^{-\sum_{j}\left(v_{j}+2 \pi k_{j}\right)^{2} / M} d \boldsymbol{v} e^{-\sum_{j} u_{j}^{2} / M} d \boldsymbol{u} \\
\ll M^{J / 2} \int_{\mathbb{R}^{J}} \int_{[0,2 \pi]^{J}}|\log | \sum_{j \leq J} b_{j} e^{u_{j}+i v_{j}}||^{2 k} d \boldsymbol{v} e^{-\sum_{j} u_{j}^{2} / M} d \boldsymbol{u} .
\end{aligned}
$$


Next we need the inequality

$$
\int_{0}^{2 \pi}\left(\log \left|a-b e^{i v}\right|\right)^{2 k} d v \ll\left(C_{1} \log |a|\right)^{2 k}+\left(C_{1} \log |b|\right)^{2 k}+\left(C_{1} k\right)^{2 k}
$$

for some constant $C_{1}>0$. (See Lemma 2.1 in [4] for a proof.) Hence, we see that

$$
\begin{aligned}
& \int_{\mathbb{R}^{J}} \int_{[0,2 \pi]^{J}}|\log | \sum_{j \leq J} b_{j} e^{u_{j}+i v_{j}}||^{2 k} d \boldsymbol{v} e^{-\sum_{j} u_{j}^{2} / M} d \boldsymbol{u} \\
& \ll \int_{\mathbb{R}^{J}}\left(\sum_{j \leq J}\left(C_{2} \log \left|b_{j} e^{u_{j}}\right|\right)^{2 k}+\left(C_{2} k\right)^{2 k}\right) e^{-\sum_{j} u_{j}^{2} / M} d \boldsymbol{u} \\
& \ll \sum_{j \leq J} \int_{\mathbb{R}^{J}}\left(\left(C_{3} u_{j}\right)^{2 k}+C_{3}^{2 k}\right) e^{-\sum_{j} u_{j}^{2} / M} d \boldsymbol{u}+M^{J / 2}\left(C_{2} k\right)^{2 k} \\
& \ll M^{J / 2} \sum_{j \leq J} \int_{\mathbb{R}^{J}}\left(\left(C_{3} u_{j}\right)^{2 k} M^{k}+C_{3}^{2 k}\right) e^{-\sum_{j} u_{j}^{2}} d \boldsymbol{u}+M^{J / 2}\left(C_{2} k\right)^{2 k} \\
& \ll M^{J / 2}\left(C_{4} k M\right)^{k}+M^{J / 2}\left(C_{2} k\right)^{2 k} .
\end{aligned}
$$

Thus,

$$
\int_{\mathbb{R}^{2 J}}|\log | \sum_{j \leq J} b_{j} e^{u_{j}+i v_{j}}||^{2 k} e^{-\sum_{j}\left(u_{j}^{2}+v_{j}^{2}\right) / M} d \boldsymbol{u} d \boldsymbol{v} \ll M^{J+k}(C k)^{k}+M^{J}(C k)^{2 k} .
$$

3.4. Proof of Proposition 2.4. By symmetry, it is enough to estimate

$$
\mathcal{E}_{\boldsymbol{m}, \boldsymbol{n}, 1}(\theta, T):=\int_{\mathbb{R}^{J}} \int_{\mathcal{R}_{1}} \log \left|1+\sum_{j=2}^{J} \frac{b_{j}}{b_{1}} e^{\left(u_{j}-u_{1}+i\left(v_{j}-v_{1}\right)\right) \sqrt{\theta \log \log T}}\right| e^{-\sum_{j}\left(u_{j}^{2}+v_{j}^{2}\right) / \xi_{j}} \boldsymbol{u}^{\boldsymbol{m}} \boldsymbol{v}^{\boldsymbol{n}} d \boldsymbol{u} d \boldsymbol{v} .
$$

Let $A_{T}=(\log \log \log T) / 4$. We divide $\mathcal{R}_{1}$ into a disjoint union of the sets:

$$
\begin{aligned}
\mathcal{R}_{1, S}:=\left\{\left(u_{1}, \ldots, u_{J}\right) \in \mathcal{R}_{1}:-\right. & \frac{A_{T}}{\sqrt{\theta \log \log T}}<u_{\ell}-u_{1} \leq 0 \text { for } \ell \in S, \\
& \left.u_{j}-u_{1} \leq-\frac{A_{T}}{\sqrt{\theta \log \log T}} \text { for } j \in\{2, \ldots, J\} \backslash S\right\}
\end{aligned}
$$

for $S \subset\{2, \ldots, J\}$. Let

$$
\begin{aligned}
\mathcal{E}_{S} & :=\mathcal{E}_{\boldsymbol{m}, \boldsymbol{n}, 1, S}(\theta, T) \\
& :=\int_{\mathbb{R}^{J}} \int_{\mathcal{R}_{1, S}} \log \left|1+\sum_{j=2}^{J} \frac{b_{j}}{b_{1}} e^{\left(u_{j}-u_{1}+i\left(v_{j}-v_{1}\right)\right) \sqrt{\theta \log \log T}}\right| e^{-\sum_{j}\left(u_{j}^{2}+v_{j}^{2}\right) / \xi_{j}} \boldsymbol{u}^{\boldsymbol{m}} \boldsymbol{v}^{\boldsymbol{n}} d \boldsymbol{u} d \boldsymbol{v},
\end{aligned}
$$

so that

$$
\mathcal{E}_{\boldsymbol{m}, \boldsymbol{n}, 1}(\theta, T)=\sum_{S \subset\{2, \ldots, J\}} \mathcal{E}_{S}
$$

First consider $\mathcal{E}_{\emptyset}$. In this case it is easy to see that

$$
\mathcal{E}_{\emptyset}=\int_{\mathbb{R}^{J}} \int_{\mathcal{R}_{1, \emptyset}} O\left(e^{-A_{T}}\right) e^{-\sum_{j}\left(u_{j}^{2}+v_{j}^{2}\right) / \xi_{j}} \boldsymbol{u}^{m} \boldsymbol{v}^{\boldsymbol{n}} d \boldsymbol{u} d \boldsymbol{v}=O\left(e^{-A_{T}}\right) .
$$


Next consider $S \neq \emptyset$, then there is at least one element $\ell \in S$. We first observe the $u_{\ell}$ integral:

$$
\begin{aligned}
& \int_{u_{1}-\frac{A_{T}}{\sqrt{\theta \log \log T}}}^{u_{1}} \log \left|1+\sum_{j=2}^{J} \frac{b_{j}}{b_{1}} e^{\left(u_{j}-u_{1}+i v_{j}-i v_{1}\right) \sqrt{\theta \log \log T}}\right| e^{-u_{\ell}^{2} / \xi_{\ell}} u_{\ell}^{m_{\ell}} d u_{\ell} \\
& \ll \int_{-\frac{A_{T}}{\sqrt{\theta \log \log T}}}^{0}|\log | \frac{b_{\ell}}{b_{1}} e^{\left(u_{\ell}+i v_{\ell}-i v_{1}\right) \sqrt{\theta \log \log T}}+\sum_{j \neq \ell} \frac{b_{j}}{b_{1}} e^{\left(u_{j}-u_{1}+i v_{j}-i v_{1}\right) \sqrt{\theta \log \log T}}|| d u_{\ell} \\
& \ll \frac{1}{\sqrt{\log \log T}} \int_{e^{-A_{T}}}^{1}|\log | \frac{b_{\ell}}{b_{1}} w e^{i\left(v_{\ell}-v_{1}\right) \sqrt{\theta \log \log T}}+\sum_{j \neq \ell} \frac{b_{j}}{b_{1}} e^{\left(u_{j}-u_{1}+i v_{j}-i v_{1}\right) \sqrt{\theta \log \log T}}|| \frac{d w}{w} \\
& \ll \frac{A_{T}}{\sqrt{\log \log T}}+\frac{1}{\sqrt{\log \log T}} \int_{e^{-A_{T}}}^{1}|\log | w+\sum_{j \neq \ell} \frac{b_{j}}{b_{\ell}} e^{\left(u_{j}-u_{1}+i v_{j}-i v_{\ell}\right) \sqrt{\theta \log \log T}}|| \frac{d w}{w} \\
& \ll \frac{e^{A_{T}}}{\sqrt{\log \log T}}
\end{aligned}
$$

by the substitution $w=e^{u_{\ell} \sqrt{\theta \log \log T}}$. Here, the last inequality holds by the following lemma.

Lemma 3.1. Let $B$ be a fixed positive real number and let $\epsilon_{T}>0$ be a decreasing function to 0 as $T \rightarrow \infty$. Then we have

$$
\int_{\epsilon_{T}}^{1}|\log | u+z|| \frac{d u}{u}=O\left(\frac{1}{\epsilon_{T}}\right)
$$

as $T \rightarrow \infty$ uniformly for all $|z| \leq B$.

Proof. We first observe that it is enough to prove that

$$
\int_{0}^{1}|\log | u+z|| d u=O(1)
$$

uniformly for bounded $z=\alpha+i \beta$. By the inequality

$$
\begin{gathered}
-|\log | u+\alpha|| \leq \log |u+\alpha| \leq \log |u+z|=\log \sqrt{(u+\alpha)^{2}+\beta^{2}} \leq \log \sqrt{2 \max \left\{(u+\alpha)^{2}, \beta^{2}\right\}} \\
=\log \sqrt{2}+\max \{\log |u+\alpha|, \log |\beta|\} \leq \log \sqrt{2}+|\log | u+\alpha||+\log B,
\end{gathered}
$$

we see that

$$
\int_{0}^{1}|\log | u+z|| d u \leq \int_{0}^{1}|\log | u+\alpha \| d u+O(1) .
$$

If $|\alpha| \geq 2$, then it is easy to see that

$$
\int_{0}^{1}|\log | u+\alpha|| d u=\int_{0}^{1}|\log | \alpha|+O(1)| d u=O(1) .
$$

If $|\alpha|<2$, then we split the interval into two intervals depending on the condition $\log |u+\alpha| \geq$ 0 . Thus,

$$
\int_{0}^{1}|\log | u+\alpha|| d u=\int_{|u+\alpha| \geq 1} \log |u+\alpha| d u+\int_{|u+\alpha|<1}-\log |u+\alpha| d u .
$$


It is easy to see that

$$
0 \leq \int_{[0,1] \cap\{|u+\alpha| \geq 1\}} \log |u+\alpha| d u \leq \log (1+B)
$$

and

$$
0 \leq \int_{[0,1] \cap\{|u+\alpha|<1\}}-\log |u+\alpha| d u \leq 2 \int_{0}^{1}-\log u d u \leq 2 .
$$

Hence, we find that

$$
\mathcal{E}_{S}=O\left(\frac{e^{A_{T}}}{\sqrt{\log \log T}}\right)
$$

for $S \neq \emptyset$ and

$$
\mathcal{E}_{\boldsymbol{m}, \boldsymbol{n}, 1}(\theta, T)=O\left(e^{-A_{T}}\right)+O\left(\frac{e^{A_{T}}}{\sqrt{\log \log T}}\right)=O\left(\frac{1}{(\log \log T)^{1 / 4}}\right) .
$$

3.5. Proof of Lemma 2.5. We see that for a fixed real $\beta$ and for each $i=1,2$

$$
\theta_{1}^{\beta}-\theta_{2}^{\beta}=H_{T}\left(\beta \theta_{i}^{\beta-1}+O\left(H_{T}\right)\right) .
$$

Thus, by (2.5)

$$
\begin{aligned}
\theta_{1}^{\alpha} I_{\boldsymbol{m}, \boldsymbol{n}}\left(\theta_{1}, T\right) & -\theta_{2}^{\alpha} I_{\boldsymbol{m}, \boldsymbol{n}}\left(\theta_{2}, T\right) \\
= & H_{T} \sqrt{\log \log T} d_{\boldsymbol{n}}\left(\alpha+\frac{1}{2}\right) \theta_{i}^{\alpha-1 / 2} \sum_{\ell=1}^{J} \int_{\mathcal{R}_{\ell}} u_{\ell} e^{-\sum_{j} u_{j}^{2} / \xi_{j}} \boldsymbol{u}^{\boldsymbol{m}} d \boldsymbol{u} \\
& +H_{T} d_{\boldsymbol{n}} \alpha \theta_{i}^{\alpha-1} \sum_{\ell=1}^{J} \int_{\mathcal{R}_{\ell}} \log \left|b_{\ell}\right| e^{-\sum_{j} u_{j}^{2} / \xi_{j}} \boldsymbol{u}^{m} d \boldsymbol{u} \\
& +\theta_{1}^{\alpha} \mathcal{E}_{\boldsymbol{m}, \boldsymbol{n}}\left(\theta_{1}, T\right)-\theta_{2}^{\alpha} \mathcal{E}_{\boldsymbol{m}, \boldsymbol{n}}\left(\theta_{2}, T\right)+O\left(H_{T}^{2} \sqrt{\log \log T}\right)
\end{aligned}
$$

for each $i=1,2$. Recall that $\mathcal{E}_{\boldsymbol{m}, \boldsymbol{n}}(\theta, T):=\sum_{\ell=1}^{J} \mathcal{E}_{\boldsymbol{m}, \boldsymbol{n}, \ell}(\theta, T)$. Hence, without loss of generality, we consider

$$
\begin{aligned}
& \theta_{1}^{\alpha} \mathcal{E}_{\boldsymbol{m}, \boldsymbol{n}, 1}\left(\theta_{1}, T\right)-\theta_{2}^{\alpha} \mathcal{E}_{\boldsymbol{m}, \boldsymbol{n}, 1}\left(\theta_{2}, T\right) \\
& =\int_{\mathbb{R}^{J}} \int_{\mathcal{R}_{1}} \log \left|1+\sum_{j=2}^{J} \frac{b_{j}}{b_{1}} e^{\left(u_{j}-u_{1}+i\left(v_{j}-v_{1}\right)\right) \sqrt{\log \log T}}\right| \\
& \quad \times\left(\theta_{1}^{\alpha^{\prime}} e^{-\sum_{j}\left(u_{j}^{2}+v_{j}^{2}\right) /\left(\theta_{1} \xi_{j}\right)}-\theta_{2}^{\alpha^{\prime}} e^{-\sum_{j}\left(u_{j}^{2}+v_{j}^{2}\right) /\left(\theta_{2} \xi_{j}\right)}\right) \boldsymbol{u}^{\boldsymbol{m}} \boldsymbol{v}^{\boldsymbol{n}} d \boldsymbol{u} d \boldsymbol{v}
\end{aligned}
$$

where

$$
\alpha^{\prime}=\alpha-J-\frac{\mathcal{K}(\boldsymbol{m}+\boldsymbol{n})}{2} .
$$

We see that

$$
\begin{aligned}
\theta_{1}^{\alpha^{\prime}} e^{-\sum_{j}\left(u_{j}^{2}+v_{j}^{2}\right) /\left(\theta_{1} \xi_{j}\right)} & -\theta_{2}^{\alpha^{\prime}} e^{-\sum_{j}\left(u_{j}^{2}+v_{j}^{2}\right) /\left(\theta_{2} \xi_{j}\right)} \\
= & \int_{\theta_{2}}^{\theta_{1}} \frac{\partial}{\partial w}\left(w^{\alpha^{\prime}} e^{-\sum_{j}\left(u_{j}^{2}+v_{j}^{2}\right) /\left(w \xi_{j}\right)}\right) d w
\end{aligned}
$$




$$
\begin{aligned}
& =\int_{\theta_{2}}^{\theta_{1}}\left(\sum_{j \leq J} \frac{u_{j}^{2}+v_{j}^{2}}{w^{2} \xi_{j}}+\frac{\alpha^{\prime}}{w}\right) w^{\alpha^{\prime}} e^{-\sum_{j}\left(u_{j}^{2}+v_{j}^{2}\right) /\left(w \xi_{j}\right)} d w \\
& \ll H_{T}\left(\sum_{j \leq J}\left(u_{j}^{2}+v_{j}^{2}\right)+1\right) e^{-\sum_{j}\left(u_{j}^{2}+v_{j}^{2}\right) /\left(\theta_{2} \xi_{j}\right)} .
\end{aligned}
$$

Thus, by adapting the proof of Proposition 2.4 we find that

$$
\begin{aligned}
\theta_{1}^{\alpha} \mathcal{E}_{\boldsymbol{m}, \boldsymbol{n}, 1}\left(\theta_{1}, T\right)-\theta_{2}^{\alpha} \mathcal{E}_{\boldsymbol{m}, \boldsymbol{n}, 1}\left(\theta_{2}, T\right) \\
\ll H_{T} \int_{\mathbb{R}^{J}} \int_{\mathcal{R}_{1}}|\log | 1+\sum_{j=2}^{J} \frac{b_{j}}{b_{1}} e^{\left(u_{j}-u_{1}+i\left(v_{j}-v_{1}\right)\right) \sqrt{\log \log T}}|| e^{-\sum_{j}\left(u_{j}^{2}+v_{j}^{2}\right) /\left(\theta_{2} \xi_{j}\right)} \\
\quad\left(\sum_{j \leq J}\left(u_{j}^{2}+v_{j}^{2}\right)+1\right)\left|\boldsymbol{u}^{\boldsymbol{m}} \boldsymbol{v}^{\boldsymbol{n}}\right| d \boldsymbol{u} d \boldsymbol{v} \\
\ll \frac{H_{T}}{(\log \log T)^{1 / 4}}
\end{aligned}
$$

and this completes the proof of the lemma.

\section{ACKNOWLEDGEMENT}

This work was partly done while the author was visiting Tokyo Institute of Techology in December 2017 and Nagoya University in February 2018. He would like to thank Masatoshi Suzuki and Kohji Matsumoto for their support and useful comments to this project. This work has been supported by the National Research Foundation of Korea (NRF) grant funded by the Korea government(MSIP) (No. 2016R1C1B1008405).

\section{REFERENCES}

[1] V. Borchsenius and B. Jessen, Mean motions and values of the Riemann zeta function, Acta Math. 80 (1948), 97-166.

[2] E. Bombieri and D. Hejhal, On the distribution of zeros of linear combinations of Euler products, Duke Math., 80 (1995), 821-862.

[3] H. Davenport and H. Heilbronn, On the zeros of certain Dirichlet series, J. Lond. Math. Soc. 11 (1936), $181-185,307-312$.

[4] S. Gonek and Y. Lee, Zero-density estimates for Epstein zeta functions, Q. J. Math. 68, (2017), no. 2, 301-344.

[5] J. Ha and Y. Lee, The a-values of the Riemann zeta function near the critical line, J. Math. Anal. Appl. 464, (2018), 838-863.

[6] D. Hejhal, On a result of Selberg concerning zeros of linear combinations of L-functions, Internat. Math. Res. Notices, 2000.

[7] Y. Lamzouri, S. Lester and M. Radziwiłl, Discrepancy bounds for the distribution of the Riemann zetafunction and applications, to appear J. Anal. Math., arXiv:1402.6682.

[8] Y. Lee, On the zeros of Epstein zeta functions, Forum Math. 26 (2014), 1807-1836.

[9] Y. Lee, Zero-density estimates for Epstein zeta functions of class number 2 or 3, J. Korean Math. Soc. 54 (2017), 479-491.

[10] A. Selberg, Old and new conjectures and results about a class of Dirichlet series. Proceedings of the Amalfi Conference on Analytic Number Theory, 367-385, Univ. Salerno, Salerno, 1992.

[11] S.M. Voronin, The zeros of zeta-functions of quadratic forms (in Russian), Tr. Mat. Inst. Steklova 142 (1976), 135-147. 